\title{
Images littéraires et recyclage dans les manuscrits d'Andersen
}

«À utiliser selon l'humeur »

Ane Grum-Schwensen

\section{OpenEdition}

\section{Journals}

Édition électronique

URL : http://journals.openedition.org/genesis/4221

DOI : $10.4000 /$ genesis.4221

ISSN : 2268-1590

Éditeur :

Presses universitaires de Paris Sorbonne (PUPS), Société internationale de génétique artistique littéraire et scientifique (SIGALES)

\section{Édition imprimée}

Date de publication : 1 juin 2019

Pagination : 159-168

ISSN : 1167-5101

\section{Référence électronique}

Ane Grum-Schwensen, «Images littéraires et recyclage dans les manuscrits d'Andersen », Genesis [En ligne], 48 | 2019, mis en ligne le 01 juin 2020, consulté le 25 janvier 2021. URL : http:// journals.openedition.org/genesis/4221 ; DOI : https://doi.org/10.4000/genesis.4221 


\title{
"À utiliser selon l'humeur". Images littéraires et recyclage dans les manuscrits d'Andersen
}

\author{
Ane Grum-Schwensen
}

$\mathrm{P}$ our un spécialiste de Hans Christian Andersen, il va sans dire que le moindre feuillet de cet écrivain internationalement connu et apprécié doit être collecté, identifié, transcrit et décrit sous toutes ses coutures. On n'en finit pas, néanmoins, de découvrir de nouveaux manuscrits, y compris pour les œuvres les plus célèbres ${ }^{1}$. Par ailleurs, parmi le vaste matériel déjà à disposition, de nombreux documents restent encore à identifier.

Une grande partie des manuscrits d'Andersen a certes été transcrite, publiée et mise à la disposition des chercheurs et des amateurs, mais la plupart de ces documents - cela doit-il nous surprendre? - concerne l'histoire personnelle de l'auteur : journaux intimes, calendriers et correspondances. Pour les documents à caractère poétique, qu'il s'agisse de brouillons et de copies d'œuvres connues ou de poèmes laissés inachevés, l'intérêt a été jusque-là considérablement moindre. C'est un paradoxe si l'on pense que les manuscrits et la correspondance d'Andersen, détenus par la Bibliothèque royale, ont été inscrits au patrimoine mondial de l'Unesco en 1997, au registre de la «Mémoire du Monde».

Les archives littéraires d'Andersen pourraient pourtant apporter des éclaircissements sur le contexte général de son œuvre. Leur étude nous renseignerait sur les réflexions poétiques de l'auteur et sur son approche créative, notamment sur sa manière de recourir à l'image comme point de départ de l'invention et au recyclage comme stratégie d'écriture.

Le présent article n'a pour autre ambition que de montrer la richesse et l'intérêt du fonds Andersen en illustrant, à partir d'exemples tirés de ses manuscrits, quelques-unes des formes que prend chez lui le processus créatif. Nous nous attacherons en particulier à décrire la manière dont les analogies et les aphorismes sont, chez l'écrivain, réutilisés et développés dans de nouveaux contextes, selon un processus qui confère à ses œuvres le caractère du collage. Mais disons, en préambule, un mot du fonds Andersen et de son utilisation.

\section{Les collections Andersen et leur utilisation passée}

Les nombreux manuscrits littéraires d'Andersen sont dispersés dans des collections variées, de volume très variable et de contenu parfois indéterminé, où se mêlent archives personnelles et archives de la création. Bien des témoins génétiques se trouvent au dos de lettres, de factures ou d'autres supports du même genre. L'abondance de ce matériel s'explique d'abord bien sûr par la pratique d'écriture de l'auteur, qui multipliait les brouillons, croquis et mises au net.

Andersen a constitué sa collection personnelle de manuscrits à plusieurs fins. Les mises au net ont souvent été conservées dans la perspective de potentiels cadeaux. Certains manuscrits de travail constituent un réservoir d'idées disparates, d'aphorismes et de «recherches» préservées en tant que sources d'inspiration pour de futurs poèmes. D'autres encore portent des poèmes achevés ou presque, que l'auteur n'a cependant pas jugé bon de publier, mais qu'il aura conservés parce qu'il les jugeait intéressants et peut-être réutilisables. D'autres enfin témoignent de la création des œuvres publiées, à divers stades (fragments, projets, brouillons et mises au net). La majeure partie de ces écrits sont contemporains (ou postérieurs) à la publication en 1863 de Contes et Histoires et constituent la base sur laquelle s'appuiera Andersen pour écrire ses remarques sur la création de ses récits : «On m'a dit que quelques remarques sur l'origine et le développement des contes ne seraient pas sans intérêt, d'où ce qui suit ${ }^{2}$.»

1. Par exemple le manuscrit de «Tællelyset» («La Chandelle» datant du début de l'année 1820) retrouvé dans les archives royales en 2012 et la mise au net de «Psychen» (1861; en français «La Psyché») découvert dans le château de Knuthenborg en 2015. Les titres français sont donnés selon les Euvres traduites, présentées et annotées par Régis Boyer (Paris, Gallimard, 1992).

2.H.C. Andersens Eventyr [Contes de H.C. d'Andersen], vol.VI, Copenhague, Société de la langue et de la littérature danoise et Hans Reitzel, 1990, p. 3. 
On relèvera que l'essentiel de ce matériel génétique conservé provient de la dernière partie de la vie de l'auteur, à une période où, au vu de sa notoriété croissante, lui et ses proches ont pris conscience de l'intérêt de ces archives. Elles se trouvent aujourd'hui dans les collections de la Bibliothèque royale ainsi que dans les musées de la ville d'Odense et dans la maison d'Andersen. On en trouve cependant un certain nombre dans les collections d'autres institutions publiques ainsi que chez des collectionneurs privés. C'est par exemple le cas du manuscrit du conte «Sneedronningen» (1844, «La Reine des neiges»), conservé à la bibliothèque de l'Université d'Uppsala, de plusieurs manuscrits de la collection Jean Hersholt à la bibliothèque du Congrès à Washington et du manuscrit de «Psychen». Enfin, le sort de certains manuscrits est incertain; on pense à ceux de «Den lille Havfrue» (1837; «La Petite Sirène»), «Kejserens nye Klæder» (1837; «Les Habits neufs de l'empereur») et «Elverhøi » (1845; «La Butte aux elfes»), qui ont été volés dans le musée au sein même de la maison d'Andersen au cours d'un cambriolage perpétré en 1992 et à ce jour non élucidé.

En 1905, une fois la question de la succession résolue, la majeure partie des manuscrits, archives et papiers privés d'Andersen faisant partie de la collection Collinske, constituée par Edvard Collin (1808-1886), sont arrivés dans la Bibliothèque royale du Danemark. Depuis 1878 et jusqu'à nos jours, des manuscrits en provenance de différents fonds viennent enrichir la collection de la Bibliothèque royale.

En 1906, soit deux ans déjà avant de se constituer en musée, la maison d'Andersen à Odense avait commencé à rassembler et à cataloguer une petite collection de pièces importantes qui s'est continuellement enrichie de nouvelles acquisitions et de nouveaux dons.

Pour les collections de ces deux institutions, rares sont les manuscrits accessibles en ligne. Par conséquent, en l'état actuel, celui qui souhaite avoir une vue d'ensemble des archives d'Andersen ou étudier des documents non directement liés aux contes et récits doit s'adresser aux institutions de conservation ou aux collectionneurs privés. L'analyse génétique d'une œuvre particulière réclame un travail de recherche transversal, les documents concernés risquant d'être répartis sur plusieurs sites.

Certaines collections de manuscrits littéraires ont fait l'objet de publications partielles et sporadiques. Il s'agit

\section{Une sélection de manuscrits d'Andersen - un projet pilote}

Au Centre Andersen situé dans les locaux de l'Université du sud du Danemark, nous travaillons actuellement (20172019) à un projet pilote qui, en réalisant une version numérique des manuscrits de l'histoire la plus longue et la plus complexe d'Andersen, «La Vierge des glaces », constituera un modèle de numérisation pour les autres manuscrits de ses Contes et Récits.

La version bêta, en danois, peut être consultée à l'adresse suivante : http://andersen.sdu.dk/ms

Ce projet pilote est financé par le fonds danois Augustinus Fonden.

avant tout de brouillons des contes et de leur mise au net. Lorsque la Société de la langue et de la littérature danoise a publié une édition critique des Contes d'Andersen de 1963 à 1990, l'éditeur et historien de la littérature Erik Dal a conclu :

Notre travail sur ces questions a clairement montré le besoin d'une vraie édition manuscrite (avec fac-similés et transcriptions) car pour notre objectif présent [une édition critique], les manuscrits n'ont apporté qu'une aide limitée ${ }^{3}$.

Si une édition des manuscrits n'a pas été encore réalisée ce n'est pas faute d'intérêt. L'hétérogénéité et la dispersion des «archives» sont davantage à incriminer. À cela s'ajoute le coût que représente une transcription générale. Enfin, il n'est pas évident pour la communauté des spécialistes que, sur le plan analytique et philologique, il vaille la peine de se lancer dans la transcription, difficile, des nombreux manuscrits d'Andersen en écriture gothique.

Pour celui qui se penche sur les écrits d'Andersen, l'historien de la littérature Helge Topsøe-Jensen (1896-1976) est incontournable. Il est l'auteur de nombreuses publications sur Andersen, comme par exemple sa thèse d'État Le Vrai Conte de ma Vie - Une étude sur Hans Christian Andersen comme auteur d'autobiographie, 1940, ou encore l'éditeur de sa correspondance et d'une version commentée de son autobiographie posthume : L'Autobiographie et les Journaux intimes de Hans Christian Andersen, 1943. Nul chercheur n'a autant travaillé sur Andersen que Topsøe-Jensen, y compris

3. Contes d'Andersen, vol. I, Copenhague, Société de la langue et de la littérature danoise et Hans Reitzel, 1963, p. 12. 
sur les manuscrits, dont nous lui devons la plupart des transcriptions publiées (un ensemble néanmoins maigre et constitué de manière opportuniste $)^{4}$.

Pour illustrer la façon dont les études dans les «Archives littéraires d'Andersen » peuvent renouveler l'éclairage sur l'œuvre, je me concentrerai désormais sur le processus de création de l'auteur. Mes exemples montreront que la méthode génétique, de collation, d'organisation et d'analyse des documents d'archives, permet de dégager des formes de processus et des relations entre documents de différentes natures.

\section{Critique génétique - positions danoises et applications à Andersen}

Alors que la critique génétique fait partie depuis les années 1970 des sciences de la littérature dans le monde francophone, elle n'a été introduite dans le monde anglosaxon qu'à partir de 2004, grâce à la publication, dans l'anthologie Genetic Criticism - Texts and Avant-textes 5 , de plusieurs articles clés qui ont jeté les bases de la théorie. En danois, c'est Johnny Kondrup qui a présenté pour la première fois l'approche génétique dans un article de 2005 dont le titre en français serait «Tout le pouvoir aux variantes? Quelques réflexions sur la critique génétique et la nouvelle philologie ${ }^{»}$.

Dans un ouvrage de référence pour l'édition scientifique danoise, Editionsfilologi (2011), Kondrup rappelle que la critique génétique se caractérise en particulier par les deux aspects suivants : la mise au premier plan du concept de travail et l'absence de hiérarchie entre états transitoires et état final.

La philologie de l'édition moderne définira une œuvre comme la somme de ses variantes. Ceci est également le point de vue de ce livre. Cependant, il n'y a aucune raison de mettre sur un même plan les variantes comme le ferait la critique génétique?

En 2014 et 2015, dans plusieurs numéros de la revue danoise Kritik, le débat sur la hiérarchie entre les versions d'une œuvre (illustré par des exemples d'éditions génétiques numériques) a opposé Poul Behrend et Johnny Kondrup ${ }^{8}$.

Bien que la critique génétique ne soit plus aujourd'hui une terra incognita dans les cercles littéraires danois, les études génétiques publiées restent rares. On peut le regretter, car il est certain qu'un fonds comme celui d'Andersen, pour en revenir à lui, présente un grand potentiel pour de tels travaux, ainsi que pour des éditions numériques.

Les outils apportés par de Biasi dans «Towards a Science of Literature : Manuscript Analysis ${ }^{9} \gg$ se sont avérés utiles pour la spécification, la classification, le décryptage et la transcription des manuscrits de travail d'Andersen. Je pense notamment à la distinction qu'il propose, à la suite de Jean Bellemin-Noël, entre le dossier génétique, comme ensemble des documents écrits conservés qui témoignent de l'écriture d'une œuvre, et l'avant-texte, comme reconstruction de l'évolution du texte que le critique peut établir sur la base du dossier.

Dans le champ anglophone, de Biasi a continué à développer le modèle, puis Dirk van Hulle et son équipe de l'université d'Anvers ${ }^{10}$. Comme le remarque ce dernier,

4. Par exemple Helge Topsøe-Jensen, «Fra en Digters værksted H.C. Andersens optegnelsesbøger», Fund og Forskning, n 9, 1962, p. $148-186$ et $\mathrm{n}^{\circ} 10,1963$, p. 119-151.

5. J. Deppman, D. Ferrer et M. Groden (dir.), Genetic Criticism - Texts and Avant-textes, University of Pennsylvania Press, 2004.

6. Danske Studier 2005, C.A. Reitzel, 2005, p. 93-116. L'introduction de Kondrup semble critiquer en particulier les déclarations de l'un des premiers représentants de cette école, Jean Bellemin-Noël, qui privent les intentions de l'auteur et le contexte littéraire et historico-culturel de toute valeur analytique. Le lecteur de Genesis trouvera l'approche de Kondrup illustrée à propos des manuscrits de Søren Kierkegaard dans le numéro 34 (p. 153-185).

7. Johnny Kondrup, Editionsfilologi, Copenhague, Museum Tusculanum, 2011, p. 193.

8. Voir notamment Poul Behrend, «Genetisk kritik», Kritik, n² 214 Copenhague, Gyldendalske Boghandel, 2015, p. 152-171, et Johnny Kondrup, «Afsluttende replik», Kritik, $\mathrm{n}^{\circ} 215$, Copenhague, Gyldendalske Boghandel, 2015, p. 193-194.

9. Pierre-Marc de Biasi, «Toward a Science of Literature : Manuscript Analysis » dans Genetic Criticism - Texts and Avant-textes, op. cit., p. 36-68 [« Vers une science de la littérature: l'analyse de manuscrits et la genèse de l'œuvre», Encyplodedia Universalis : Symposium, Paris, 1985, p. 466-476].

10. Pierre-Marc de Biasi, «What is a literary draft? Toward a functional typology of genetic documentation », Yale French Studies, no 89, 1996, p. 26-58 [ Édition horizontale, édition verticale. Pour une typologie des éditions génétiques (le domaine français 1980-1995)», dans B. Didier, J. Neefs (dir.), Éditer des manuscrits. Archives, complétude, lisibilité, Presses universitaires de Vincennes, «Manuscrits modernes», 1996]. Dirk van Hulle, «Modelling a Digital Scholarly Edition for Genetic Criticism: A Rapprochement», Variants - The Journal of the European Society for Textual Scholarship, $\mathrm{n}^{\circ} 12-13$, p. 34-56. 
«a writer's creative process is often an interaction with books and notebooks » [le processus créatif de l'auteur est souvent une interaction avec des livres et des cahiers] ${ }^{11}$. Ceci est vrai quand on aborde les travaux préparatoires d'Andersen. Pour cette raison, lors de l'élucidation des modèles créatifs que j'ai proposés dans ma thèse de doctorat (Fra Strøtanke til vark [De la pensée parcellaire à l'œuvre], 2014) et que j'illustrerai plus loin, en plus des brouillons de l'auteur portant spécifiquement sur l'œuvre en cours d'élaboration, mon analyse intégrait d'autres manuscrits de l'auteur, au titre de ce que nous pourrions appeler des références intertextuelles internes (des notes de carnets et des lettres), ainsi qu'un certain nombre de références intertextuelles externes ${ }^{12}$. Mon analyse du processus créatif se situe ainsi à la frontière de l'exogenèse et de l'endogenèse, en incluant des textes qui ont indéniablement un caractère privé, mais qui contiennent néanmoins du matériel verbal, narratif ou descriptif, devant être considéré comme une condition préalable à la production destinée au public.

\section{La pratique créative d'Andersen}

Présentons tout d'abord une collection hétérogène et volumineuse, et cependant négligée, des dossiers et projets d'Andersen : la collection Collinske 41, 4o. Le catalogue de la Bibliothèque royale la décrit ainsi :

Livrets et documents volants divers, y compris notes éparses, brouillons, extraits, etc., parties de A. rassemblés sous le terme «pensées et notes à utiliser selon l'humeur» (Au total 467 feuillets décrits) ${ }^{13}$.

Ces «feuilles volantes » ont été rassemblées en plusieurs groupes par Andersen lui-même. On trouve dans cette collection quatre couvertures : deux d'entre elles portent le titre «Pensées et notes à utiliser selon l'humeur», une troisième contient des coupures de journaux et s'intitule «Poèmes et notes à utiliser selon l'humeur» (fig. 1) tandis que la quatrième fait à l'évidence référence à deux catégories différentes de documents : «Mes propres strophes manuscrites, ne conviennent pas. Manuscrits étrangers. » Il s'agit d'une collection importante. En comparant par exemple le nom de cette collection 41, 40 avec celui de la collection Collinske 36, 40 : «Contes et histoires I-V + Registre.

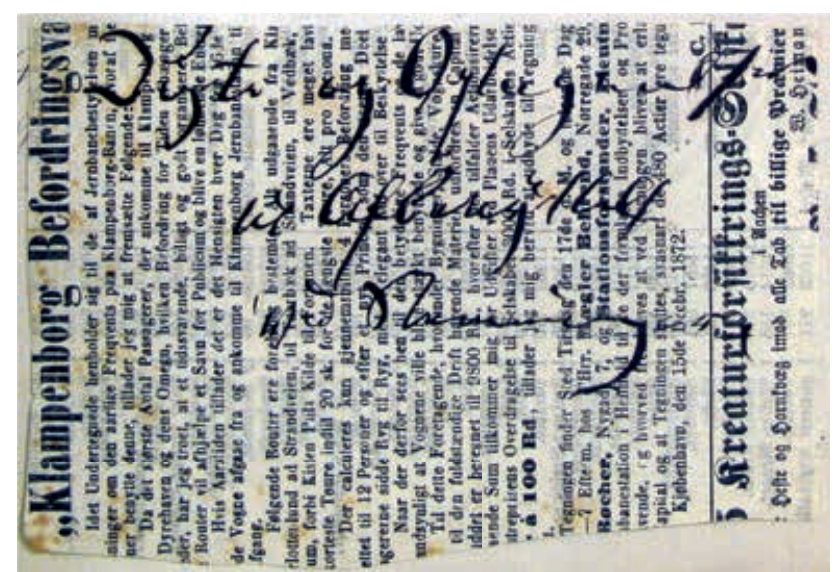

Fig. $1:$ «Digte og Optegnelser til Afbenyttelse ved Stemning» [« Poèmes et notes à utiliser selon l'humeur»]. Une des nombreuses couvertures présentes dans la collection Collinske 41, 40. Enregistrement privé, Bibliothèque royale, Copenhague.

Brouillons, projets ou mises au net», on comprend combien il peut être difficile de distinguer a priori les brouillons de ces deux ensembles.

La collection Collinske 41, 40 apparaît comme un trésor lorsqu'on étudie ce que l'on pourrait nommer «les exercices initiaux » de l'auteur. Elle est constituée d'un grand nombre de brouillons, fragments et autres projets «terminés» sous forme de feuilles volantes, en plus de cinq livrets qui contiennent à la fois des recherches préliminaires, de vrais brouillons ainsi que des recueils d'aphorismes (cités ou inventés) et d'analogies.

Cet ensemble fait d'Andersen un poète économe qui collectait constamment ses trouvailles. Lorsqu'il avait trouvé, «selon l'humeur», une utilisation pour ses fragments, il les marquait soigneusement d'une croix. Avec

11. «Modelling a Digital Scholarly Edition», art. cit., p. 36. 12. Ane Grum-Schwensen, Fra strøtanke til vark. En genetisk unders $\phi$ gelse af de kreative processer $i$ den sene del af H.C. Andersens forfatterskab, thèse de doctorat, 2014. Un résumé en anglais est téléchargeable sur le site de l'Université du sud du Danemark < http://findresearcher.sdu.dk/ portal/da/publications/fra-stroetanke-til-vaerk(f26f37a5-5667-4472bc4d-d5ac2542cb50).html >.

13. Lauritz Nielsen, Katalog over danske og norske Digteres Originalmanuskripter $i$ Det Kongelige Bibliotek, Copenhague, Munksgaard, 1943, p. 12. 
ces «exercices préliminaires » en tête, on est conduit à reconsidérer l'image de l'écrivain à écriture spontanée qu'il a cultivée en son temps et qui a perduré depuis. Même Topsøe-Jensen, qui montre dans ses travaux la complexité des processus scripturaux chez Andersen, en propose une synthèse quelque peu trompeuse :

Normalement, un conte est écrit en l'espace de quelques jours. Certains des plus célèbres tels que «Les Galoches du bonheur», «Le Vilain Petit Canard», «La Reine des neiges », et «L'Ombre» ont une genèse plus longue ${ }^{14}$.

Les notes issues des carnets sont souvent les premiers éléments du dossier à constituer. Si l'on consulte les journaux intimes et les lettres de l'auteur, on trouve souvent des idées et des pensées qui peuvent être considérées comme appartenant déjà au processus de création se concrétisant dans l'œuvre. Il est intéressant de remarquer que ces premières notes associables à une œuvre publiée ont la forme d'images linguistiques telles que des analogies ou des métaphores, mais aussi des prosopopées ou des aphorismes.

Ces matériaux verbaux qui constituent les premiers témoins de l'avant-texte offrent un aperçu de la manière dont l'auteur incorpore des matières textuelles hétérogènes au cours du processus. C'est aussi le cas d'emprunts intertextuels (à sa propre œuvre ou à d'autres) dont le repérage n'est possible, le plus souvent, qu'en comparant les textes publiés. L'analyse de ces emprunts, comme processus d'intégration du déjà-écrit, touche à un aspect essentiel de la méthode d'Andersen. Et il est particulièrement frappant de noter que ces phénomènes d'intégration intertextuelle apparaissent tardivement dans le processus d'écriture.

L'intertextualité nous conduit à un phénomène connexe caractéristique des œuvres publiées par Andersen, mais peut-être encore plus évident lorsque l'on travaille sur ses manuscrits : l'utilisation récurrente de thèmes particuliers (l'opposition entre essence et apparence, la mortalité, la littérature à sauver des poubelles de la mémoire...), ainsi que le retour des mêmes analogies ou aphorismes dont nous avons déjà parlé. L'auteur tend à recycler son matériel littéraire.

Une autre caractéristique de l'écriture chez Andersen est l'activité de collage comme principe de composition. Le mot doit s'entendre à la fois en un sens abstrait, décrivant différents procédés de reproduction de fragments prenant souvent la forme de récits enchâssés, et au sens purement concret, comme le montre la figure 5. On en verra plus loin des exemples - à partir de documents tirés des dossiers de «Iisjomfruen» (1861; «La Vierge des glaces») et «Tante Tandpine» (1872; «Tante Mal-aux-Dents ») - qui aideront à comprendre dans quel sens on peut parler de phénomènes de recyclage et de collage chez Andersen 15 .

\section{L'image comme point de départ de la création}

Lorsque j'évoque l'image en tant que point de départ du processus créatif chez Andersen, je pense tout d'abord à l'image linguistique, c'est-à-dire à différents types de comparaisons que l'on trouve en quantité dans de nombreux cahiers de notes et fragments figurant dans la collection Collinske 41, 40 ainsi que dans les notes des journaux intimes.

Dans les notes des cahiers utilisés par l'auteur pour «Tante Tandpine», on trouve un grand nombre d'analogies de ce genre16:

La femme fait un rêve au cours duquel elle perd toutes ses dents. «Maintenant je perds un de mes amis !» dit-elle. «Oh, ce n'est pas un vrai ami, toutes les dents sont fausses 17 !»

Les notes, prises dans un petit cahier comprenant trente pages non numérotées, datent du début des années 1850 et témoignent que l'auteur avait déjà, vingt ans avant la première publication de «Tante Tandpine», pensé à utiliser cette analogie humoristique en mettant en scène, comme dans l'histoire finale, une femme ayant de fausses dents.

14. Helge Topsøe-Jensen, Buket til Andersen, Copenhague, G.E.C. Gad, 1971, p. 275.

15. La majeure partie de ce matériel a été publiée pour la première fois en annexe de mon mémoire de troisième cycle : Fra strøtanke til vark, op.cit., disponible en ligne : < https://portal.findresearcher.sdu.dk/ files/116720882/Ane_Grum_Schwensen_afhandling.pdf >. Le conservateur en chef du Musée de la ville d'Odense, Ejnar Stig Askgaard a effectué les transcriptions.

16. En annexe à la thèse précédemment mentionnée, on trouve un aperçu complet des éléments figurant dans le dossier utilisé pour «Tante Tandpine» et Iisjomfruen.

17. Note $\mathrm{n}^{\circ} 12$ du cahier II- 1 constitué de 30 pages non numérotées. Ce cahier a été publié, dans une transcription peu fidèle de Julius Clausen, en 1926 sous le titre Livret de notes de H.C. Andersen. 
Dans la mise au net ${ }^{18}$, l'analogie est développée comme suit (fig. 2) :

Un matin, à table, au déjeuner, elle raconte un rêve sinistre qu'elle a eu au cours de la nuit: elle a perdu une de ses dents.

Elle explique «Cela signifie que j'ai perdu un(e) vrai(e) $\operatorname{ami}(\mathrm{e})$ !»

«C'était une fausse dent! » dit le brasseur, «et cela ne peut avoir qu'une seule explication. Vous avez perdu un(e) faux(sse) ami(e)!»

Ce manuscrit manifeste le travail acharné et détaillé d'Andersen sur les choix et la formulation des mots, et sur le fait que le terme «mise au net» doit être utilisé avec précaution lorsque l'on parle d'écriture d'invention. Il est de règle que les «mises au net» d'Andersen comportent des traces de révision nombreuses et souvent importantes, témoignant de plusieurs étapes de rédaction, notamment à l'endroit des fins de phrases, qui éclairent de manière décisive le travail de l'écrivain.

Tout comme le parallélisme imagé présenté ci-dessus, un grand nombre d'analogies, de métaphores et d'aphorismes contenus dans des cahiers de notes ou sur des feuilles volantes de la collection Collinske 41, 4o peuvent être mis en lien avec des œuvres publiées. À bien des égards, on peut donc considérer ces cahiers comme un catalogue d'idées consulté au cours du travail de rédaction de l'auteur. Cela est particulièrement visible dans l'écriture de la prose poétique de «Det nye Aarhundredes Musa» (1861; «La Muse du nouveau siècle») : lors de son travail sur la dernière des trois ébauches, Andersen a repris toutes les notes à caractère poétique des cahiers en marquant celles-ci d'une croix à mesure qu'il les utilisait.

Des impulsions visuelles plus concrètes ont servi de point de départ dans le travail de rédaction de l'auteur. Dans la collection Collinske 41, 40, un petit ensemble de pièces fragmentaires utilisé pour la rédaction de «Tante Tandpine» en présente un curieux exemple (fig. 3).

Andersen s'est ici inspiré de la tache d'encre située dans le coin supérieur à gauche de la feuille - c'est le genre de taches qui surviennent lorsque l'on vient de plonger sa plume dans l'encrier et qu'on la garde immobile au-dessus du papier. Cela correspond à un moment d'hésitation, avant de poser la plume sur le papier et d'écrire.

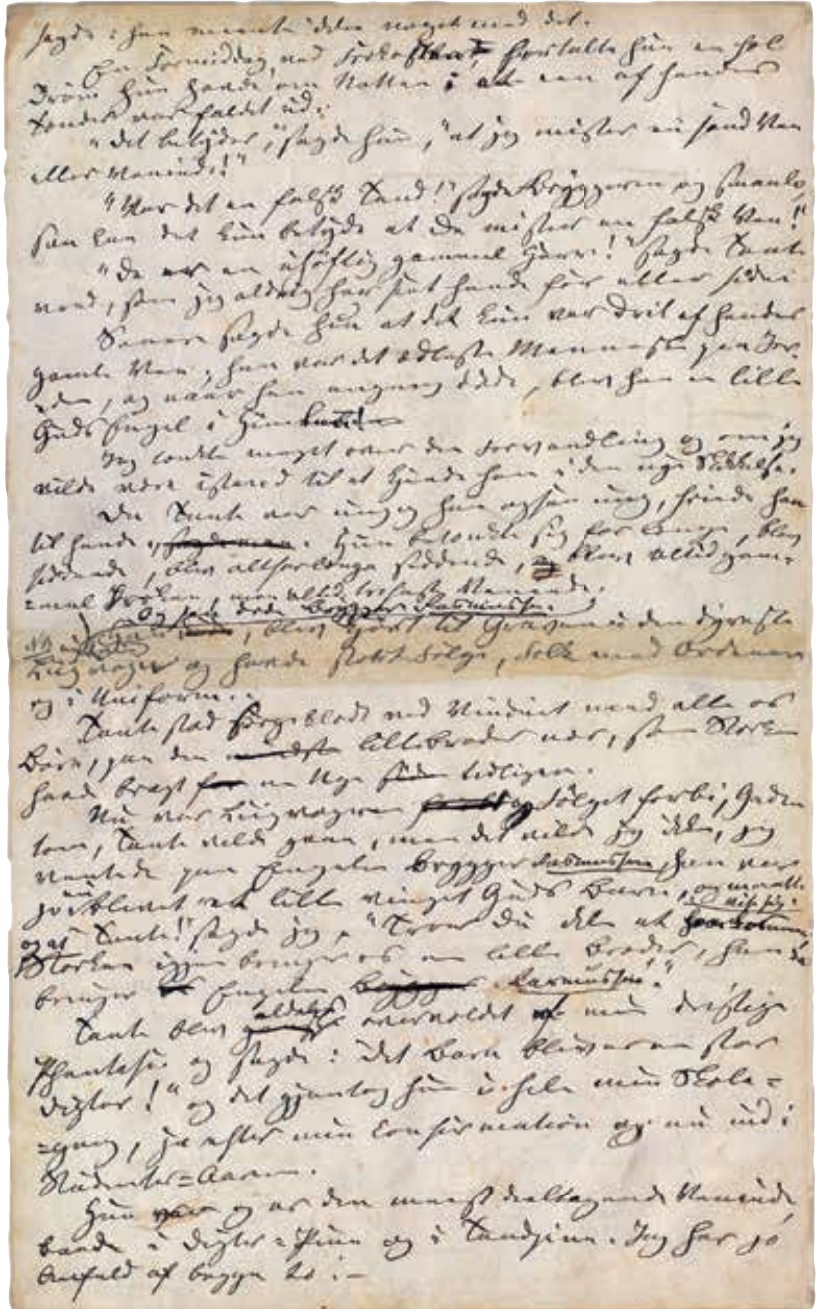

Fig. 2 : Mise au net de «Tante Tandpine» («Tante Mal-aux-Dents»), p. 4. Le récit de rêve, associant la perte de dents à celles d'amis, se situe au sommet de la page. Bibliothèque royale, Copenhague.

L'écriture peut puiser son inspiration dans la tache d'encre accidentelle dont elle parle et décrit en détail un jeu de société très répandu à l'époque (fig. 4). En même temps, se trouve développée une analogie entre le jeu en question et le travail du poète, avançant ainsi une hypothèse sur le rôle du talent et du hasard dans la conception artistique. La «poésie fruit du hasard» est un topos, et Klaus P. Mortensen explique, dans un ouvrage qui porte ce titre, ce qu'il en est pour la poétique d'Andersen ${ }^{19}$. Aussi est-il

18. La mise au net se trouve dans la collection Collinske 36, 4o (IV : 128). 19. Klaus P. Mortensen, Tilfaeldets poesi. H.C. Andersens forfatterskabindblik og oversigt, Copenhague, Gyldendal, 2007. 


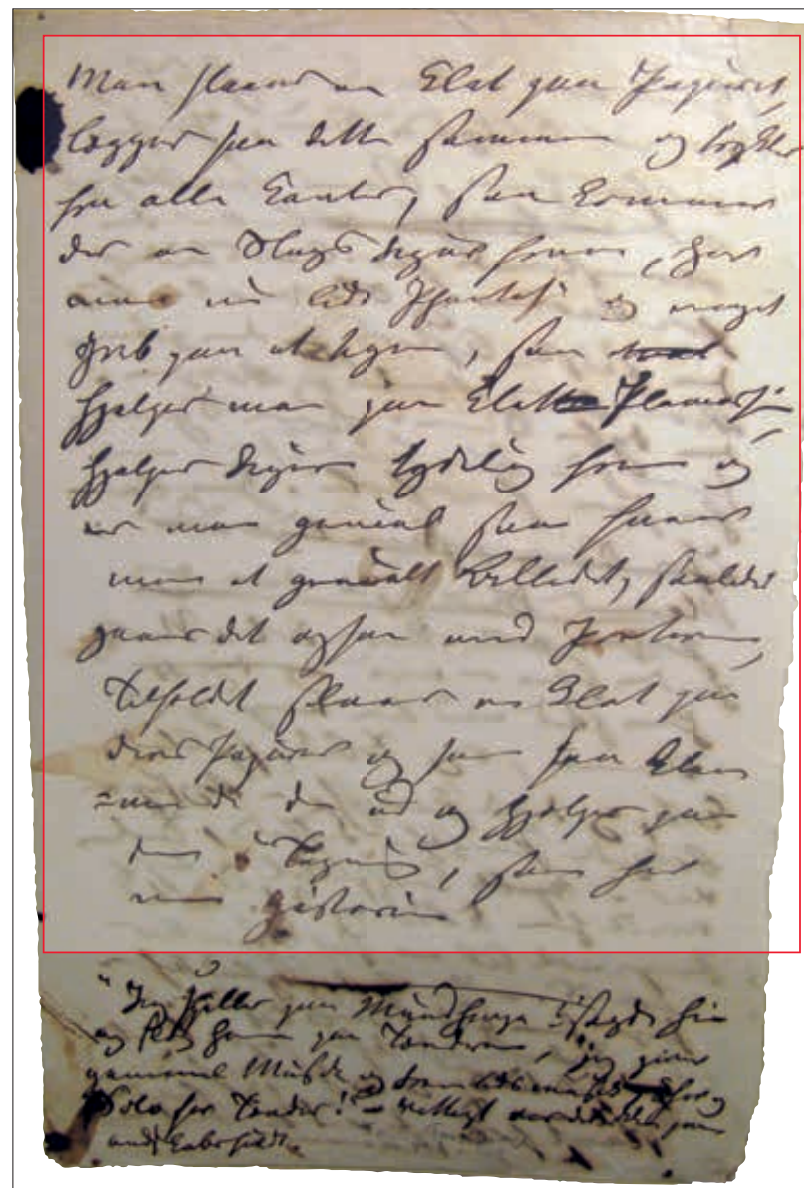

Fig. 3 : Collection Collinske 41, 4o, VI-69-a, recto. Pièce du projet fragmentaire de «Tante Tandpine», note privée. Bibliothèque royale, Copenhague.

\section{TRANSCRIPTION (texte encadré de rouge)}

On fait un point sur le papier, puis on le plie et on le presse, et puis arrive une sorte de figure. Si vous avez un peu d'imagination et si vous êtes capable de dessiner, alors $\mathrm{xxx}$ on aide la tache* et la pousse à prendre une forme plus claire, et si l'on est un génie, alors on obtient une image ingénieuse. Il en va de même avec les poètes, Chance fait un point sur leur papier et voici qu'ils le pressent et l'aident en dessinant, puis vous avez une histoire.

$(*)$ «Plamassie», dans le texte, c'est-à-dire, en danois moderne : «Plamage» (mot emprunté de l'allemand qui signifie également «honte»).

tout à fait remarquable que «Pensées et notes, feuilles volantes » (dans 41, 4o) comporte un projet de prose inédit intitulé «Poésie du hasard», dont on ne connaissait jusque-là qu'une mention de l'auteur, dans son journal, en date du 26 août 185120 .

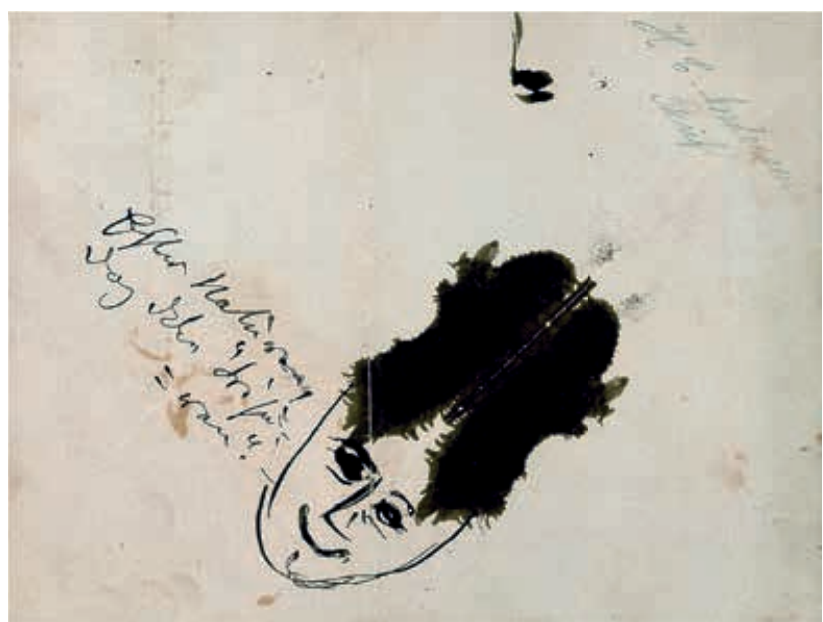

Fig. 4 : «D'après la nature, donc pas "Coiffure"». Dessin à l'encre bleu vert, Musée d'Andersen, Odense.

\section{Le « recyclage» comme stratégie créative}

Dans son article «Garbage and Recycling : From Literary Theme to Mode of Production» (2007), Walter Moser a introduit le «recyclage» comme un thème littéraire à part entière dans les œuvres des XIX ${ }^{\mathrm{e}}$ et $\mathrm{XX}^{\mathrm{e}}$ siècles 21 .

Le XIX ${ }^{\mathrm{e}}$ siècle a produit une abondante littérature avec des récits portant sur les déchets, sur l'intégration des déchets dans un cycle économique secondaire et sur les sujets humains en lien avec cette thématique 22 .

Moser explique comment les déchets et le «recyclage» sont devenus des thèmes littéraires. Il met en évidence également en quoi le «recyclage» peut être considéré comme une forme de production culturelle, et pas seulement comme un matériau réutilisé pour des raisons d'économie. Il déclare à cet égard :

Je ne voudrais pas exclure des utilisations plus métaphoriques du terme, par exemple lorsqu'un artiste reprend et transforme le travail (idée, histoire, genre et matériaux, etc.) d'un autre artiste $^{23}$.

L'utilisation du terme «recyclage» pour décrire une forme de production culturelle est très proche du concept d'intertextualité des structuralistes et du concept de collage

20. Fra strøtanke til vark, op. cit., p. 175 et H.C. Andersens dagbøger, op. cit., vol. IV, p. 70 .

21. Moser, Walter «Garbage and Recycling : From Literary Theme to Mode of Production», Other Voices, no 3 : 1, 2007. <http://othervoices. org/3.1/wmoser/index.php $>$.

22. Ibid., p. 2.

23. Ibid., p. 7 
dans les théories d'avant-garde 24 . On ne peut pas exclure, par ailleurs, l'emploi de ce mot pour décrire le fait qu'un artiste (comme Andersen) recycle et transforme un matériau de sa propre production et de ses propres travaux préparatoires (ce que j'appellerai à partir d'ici «intertextualité interne $\gg)$.

Moser distingue la «réutilisation », lorsqu'un objet reconnaissable et pratiquement intact prend une autre fonction (par exemple une bouteille réutilisée comme vase), du «recyclage», qui suppose que l'objet réemployé intègre et soit transformé par un nouveau cycle de production.

[...] le «recyclage», en particulier dans la sphère culturelle contient toujours l'idée de transformation et de métamorphose. Le recyclage ne remet jamais un système ou un matériau dans sa position ou son identité initiale. Dans chaque étape du recyclage culturel, on trouve une nouvelle production ou un nouveau sens à considérer 25 .

Le «recyclage» de thématiques, d'images linguistiques ou encore de formules poétiques fait manifestement partie, chez Andersen, d'une stratégie créative. L'existence de motifs favoris et récurrents n'est pas propre à l'univers artistique d'Andersen; nous le rencontrons chez de nombreux artistes. Mais chez lui, l'intensité et l'étendue du phénomène me paraissent assez exceptionnels, ainsi que l'empan entre les premières et les dernières occurrences des figures récurrentes. À se plonger dans les nombreuses transformations du texte, le chercheur se fait une idée de la manière, de l'étendue et du moment où le recyclage est intervenu dans le processus de production. Dans le cas d'Andersen, c'est comme si un recyclage très conscient d'éléments intertextuels internes s'opérait pour créer un réseau plus vaste reliant les œuvres entre elles. Ce fonctionnement devient évident lorsque par exemple, dans «les archives », on trouve une note 26 décrivant les liens réciproques existant entre les nombreux éléments utilisés par Andersen dans «La Dryade», «La Petite Sirène », «La Vierge des glaces» et «La Reine des neiges». De la même manière, le matériel contenu dans les archives de l'auteur permet de parler de «recyclage» en ce sens que les trouvailles initialement destinées à être utilisées dans un contexte donné le sont ensuite dans un autre. Dans la définition donnée ci-dessus par Moser, Andersen «recycle» par ses nombreuses reprises intertextuelles.
Chez Andersen, le recyclage littéraire est également une thématique. Moser, dans son article, mentionne Les sages réflexions du chat Murr entremêlées d'une biographie fragmentaire du maître de chapelle Johannès Kreisler présenté au hasard de feuillets arrachés (1819-1821), d'E. T. A. Hoffman, comme exemple d'ouvrage traitant du recyclage littéraire, de la dépendance du texte écrit à son support matériel. Pour retracer sa vie, le protagoniste, Kater Murr, utilise du vieux papier, déjà utilisé d'un côté et mis au rebut 27 : l'œuvre d'Hoffmann prend ainsi la forme de deux textes parallèles. Andersen connaissait Kater Murr et l'a évoqué dans son œuvre de jeunesse Fodreise fra Holmens Canal til Østpynten af Amager i Aarene 1828 og 1829 [Promenade du canal de Holmen à la pointe orientale d'Amagre]. Parallèlement, il a théorisé le «recyclage» littéraire dans le livre qui constitue son testament littéraire : «Tante Tandpine». Il s'agit de l'histoire d'un étudiant qui a jeté un manuscrit que le narrateur retrouve et raconte. Si l'on suit la genèse de cette histoire à travers les notes conservées et les ébauches fragmentaires du dossier, il apparaît qu'elle se compose de ces fragments de diverses natures que l'auteur conserve pour les recycler «selon $1^{\prime}$ humeur ${ }^{28} \gg$. On peut même aller jusqu'à dire que cette note : «On a des centres de secours en cas d'asphyxie, puissions-nous en trouver pour les auteurs oubliés 29 », que l'on retrouve dans plusieurs cahiers, ainsi que, raturée, dans l'une des ébauches de «La Muse du Nouveau siècle» (d'où elle disparaîtra), constitue l'analogie qui deviendra le pivot de «Tante Tandpine».

24. Qu'on pense à Gérard Genette (Palimpsestes. La littérature au second degré, 1982) et à Peter Bürger (Theory of the Avant-Garde, 1974).

25. Ibid., p. 6.

26. Note sur «Svindelen» («Le Vertige») dans la collection Collinske 41, 4ㅇ, (Cahier de notes II-2, feuillet 23, verso).

27. Die Lebens-Ansichten des Kater Murr nebst fragmentarischer Biographie des Kapellmeister Johannes Kreisler in zufälligen Makulaturblättern, Berlin, A. Hofmann, 1819-1821.

28. Voir Fra strøtanke til vark, op. cit., p. 116-164.

29. Carnet de notes II-2 [« Livre de notes»], feuillet 4 recto. Cette note est utilisée dans le conte «Tante Tandpine» ainsi que dans le roman Être ou ne pas être (1857) dont il n'existe pas de traduction française. 


\section{«Archives d'Andersen», potentiels et défis}

Plusieurs autres œuvres d'Andersen peuvent, tout comme «Tante Tandpine», être considérées comme collant et recyclant plusieurs fragments hétérogènes. C'est notamment le cas d'un texte au genre hybride, «La Vierge des glaces», qui est une composition intégrant à la fois des emprunts intertextuels et des matériaux originaux (fig. 5).

Les nombreux manuscrits d'Andersen présentant de tels collages sont beaux à contempler. Mais leur transcription et l'analyse du matériel qui les compose sont un véritable défi. Certains morceaux ne se détachent pas du papier sur lequel ils sont collés, d'autres sont écrits au verso de brouillons qui ont été découpés. Il est particulièrement difficile de reconstituer les contextes desquels ont été soustraits les bouts de brouillons ainsi recyclés.

J'ai voulu proposer ici quelques considérations isolées sur la pratique créative d'Andersen à partir de ses manuscrits littéraires. Mais mon travail génétique n'est qu'une illustration des nombreux angles possibles que l'on peut adopter pour étudier le matériel à la fois volumineux et passionnant que l'on trouve dans les «archives d'Andersen». Elles contiennent, et cela est remarquable, tout ce que le poète a laissé à sa mort, survenue en août 1875. Ce qui, de son vivant, n'avait pas été envoyé sous forme de lettres à ses connaissances ou donné sous forme de manuscrit littéraire à des chasseurs d'autographes, a été retrouvé chez lui. Edvard Collin, qui a classé ce matériel avant de le transmettre à la Bibliothèque royale, a tout conservé, jusqu' aux papiers personnels qui témoignaient de la relation d'amour-haine qui l'unissait à Andersen, jusqu' aux derniers fragments rédigés au dos de factures qu'il a retrouvé dans la maison du poète. L'énorme quantité de manuscrits,

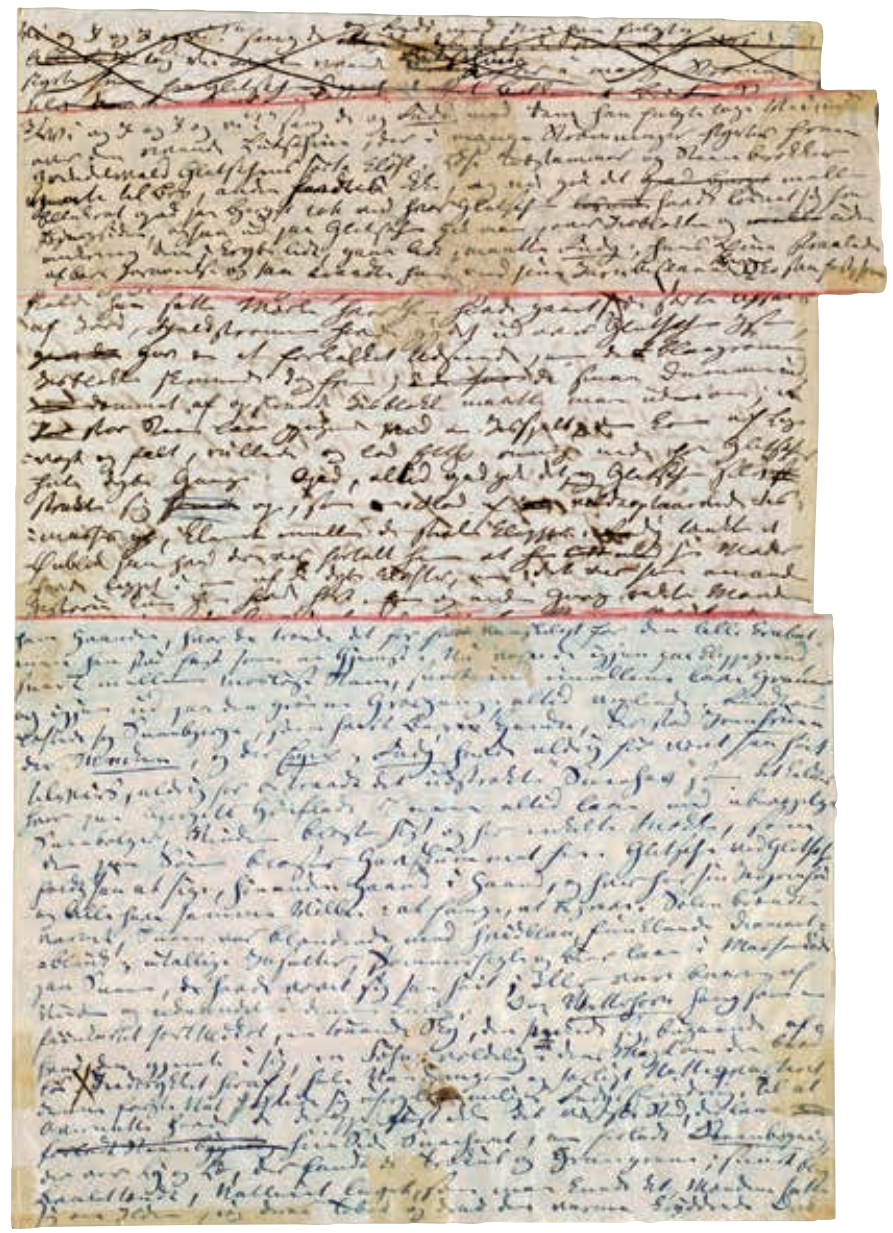

Fig. 5 : Collection Collinske 36, 4o, ébauche de «La Vierge des glaces», p. 9. Bibliothèque royale, Copenhague.

comprenant des poèmes rédigés par Andersen depuis sa vingtième année jusqu'à sa mort, est donc la plus riche des sources pour l'étude de l'écriture de l'auteur. Des archives aussi volumineuses constituent une rareté à l'échelle mondiale et l'on ne peut que regretter qu'elles n'aient pas été rendues plus accessibles. Car elles méritent et attendent de nombreuses recherches.

ANE GruM-SChWENSEN est conservatrice au musée Hans Christian Andersen de la ville d'Odense depuis 1998. Elle est également chercheuse au Centre Hans Christian Andersen de l'université du Sud Danemark. Elle a publié plusieurs articles sur l'œuvre littéraire d'Andersen et sur les collections du musée d'Andersen, et a consacré sa thèse $(\mathrm{PhD})$ aux processus créatifs dans la dernière partie de l'œuvre de l'auteur en 2014.

agrum@sdu.dk 


\section{“ À utiliser selon l'humeur ". Images littéraires et recyclage dans les manuscrits d'Andersen}

L'article présente l'héritage littéraire que nous a laissé l'auteur danois Hans Christian Andersen (1805-1875) : ses manuscrits. Ceux-ci sont principalement conservés dans deux institutions, la Bibliothèque royale de Copenhague et le musée Hans Christian Andersen d'Odense. Un projet pilote est en cours de développement au Centre Hans Christian Andersen (Université du Sud Danemark), mais un catalogue général du fonds n'existe pas encore, pas plus que des transcriptions systématiques. L'article montre comment l'organisation et l'analyse de ce matériel menées selon une approche génétique pourraient conduire à la mise au jour d'aspects importants du processus créatif chez Andersen. Si la critique génétique n'est pas très répandue dans le champ danois des recherches littéraires et textuelles, l'article dégage les positions qui s'y sont néanmoins manifestées. En plus d'une description générale de l'invention chez Andersen, la contribution explore deux voix particulières de sa pratique créative : l'image comme point de départ pour l'écriture et prérequis de son œuvre, et le recyclage comme une stratégie de création.

The article presents the literary heritage of Danish author Hans Christian Andersen (1805-75), namely his manuscripts, which are primarily preserved at two institutions: The Royal Library in Copenhagen and The Hans Christian Andersen Museum in Odense. A pilot project is presently being developed at The Hans Christian Andersen Centre, University of Southern Denmark, but an assembled and transcribed literary Andersen archive has not yet been realised. The article demonstrates how the organizing and analysing of this material with a genetic approach may lead to the uncovering of interesting characteristics of Andersen's creative process. Critique Génétique is not a widespread approach in the current Danish literary and textual research environment, but the article outlines the positions that have nevertheless manifested themselves in this regard. In addition to an overall description of the creative practise of Andersen, the article explores two specific characteristics of this practise: The image as the creative starting point and prerequisite in the authors work, and recycling as a creative strategy in Andersen's oeuvre.

Der Artikel stellt das literarische Erbe des dänischen Schriftstellers Hans Christian Andersen (1805-1875) vor, nämlich seine Manuskripte. Diese sind hauptsächlich in zwei Institutionen archiviert: in der Königlichen Bibliothek von Kopenhagen und im HansChristian-Andersen-Museum in Odense. Derzeit wird dazu am Hans Christian Andersen-Zentrum (Universität von Süddänemark) ein Pilotprojekt entwickelt. Ein vollständiger Katalog des Manuskriptbestands ist jedoch noch nicht vorhanden, ebenso wenig wie systematische Manuskript-Transkriptionen. Der vorliegende Artikel zeigt, wie die Organisation und Analyse dieses Materials mit Hilfe eines genetischen Ansatzes zur Erhellung wichtiger Aspekte von Andersens Schaffensprozess führen könnte. Obwohl die genetische Kritik in der dänischen Literaturwissenschaft nicht weit verbreitet ist, werden in diesem Artikel die Positionen dargestellt, die dennoch schon vorliegen. Neben einer allgemeinen Beschreibung von Andersens Schaffensprozess werden in diesem Beitrag zwei besondere Charakteristika seiner kreativen Praxis untersucht: Das Bild als Ausgangspunkt und Voraussetzung für sein Schreiben und das Wiederverwerten von Texten als kreative Strategie in seinem Werk.

Este artículo presenta la herencia literaria que nos ha dejado el autor danés Hans Christian Andersen (1805-1875): sus manuscritos, depositados esencialmente en dos instituciones: 1a Biblioteca Real de Copenhague y el museo Hans Christian Andersen de Odense. Un proyecto piloto está siendo desarrollado en el Centro Hans Christian Andersen (Universidad de Dinamarca del Sur), pero todavía no existe un catálogo general del fondo, ni transcripciones sistemáticas. Este artículo muestra como la organización y el análisis de este material orientados con un enfoque genético podría revelar aspectos importantes del proceso creativo en la obra de Andersen. Si bien la crítica genética no está muy difundida en el campo danés de las investigaciones literarias o textuales, este artículo puntualiza las posiciones que se están poniendo de manifiesto al respecto. Por otra parte, junto con una descripción general de la invención en Andersen, este trabajo explora dos vías particulares de su práctica creativa: la imagen como punto de partida de la escritura y prerrequisito de su obra, y el reciclado como estrategia de creación.

Este artigo faz uma apresentação do legado literário do autor dinamarquês Hans Christian Andersen (1805-1875), legado constituído pelos seus manuscritos, conservados principalmente em duas instituições: a Biblioteca Real de Copenhaga e o Museu Hans Christian Andersen, de Odense. Um projecto-piloto está sendo desenvolvido no centro dedicado ao escritor na Universidade do Sul da Dinamarca, mas não existe ainda um catálogo geral do arquivo, nem transcrições sistemáticas. A organização e a análise de tipo genético desse material poderiam levar à compreensão de aspectos importantes do processo criativo de Andersen. A crítica genética não se acha generalizada na área dos estudos literários e textuais da Dinamarca. Além de uma descrição geral dos seus processos criativos, o artigo destaca dois aspectos na obra de Andersen: a imagem como ponto de partida e pre-condição da sua escrita e a reciclagem como estratégia de criação

L'articolo presenta i manoscritti dell'autore danese Hans Christian Andersen (1805-1875), la cui eredità letteraria è conservata principalmente nella Biblioteca reale di Copenaghen e nel Museo Hans Christian Andersen di Odense. Un progetto pilota è in corso di sviluppo al centro Hans Christian Andersen dell' Università della Danimarca Meridionale (Syddansk Universitet), ma non esistono ancora né un catalogo generale del fondo né delle trascrizioni sistematiche. L'articolo mostra come l'organizzazione e l'analisi di questo materiale secondo un approccio genetico potrebbero condurre alla messa in luce di aspetti importanti del processo creativo. Se la critica genetica non è molto diffusa nel campo delle ricerche letterarie e testuali danesi, l'articolo evidenza alcune posizioni che si sono comunque manifestate. Oltre ad una descrizione generale del processo inventivo in Andersen, vi si esplorano due caratteristiche della sua pratica creativa: l'immagine come punto di partenza della scrittura e prerequisito della sua opera, e il riciclaggio come strategia di creazione. 